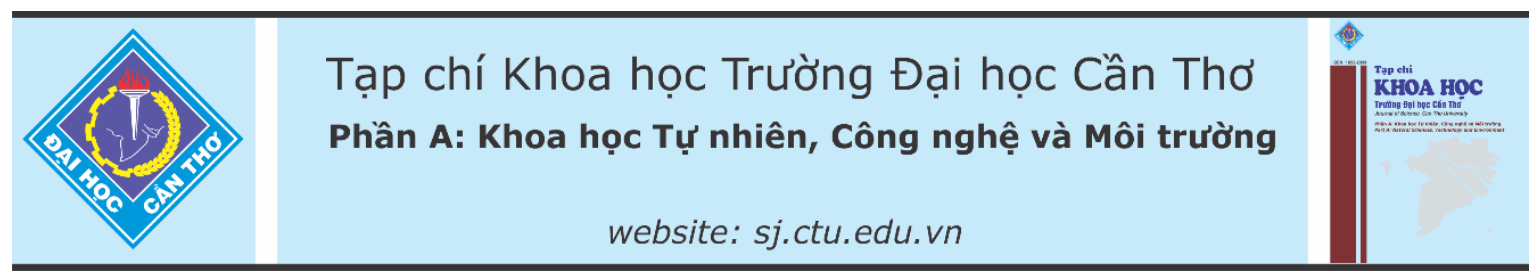

DOI:10.22144/ctu.jvn.2021.036

\title{
LUẬT SỐ LỚN TRONG MÔ HÌNH TRÒ CHƠI KHÔNG CÔNG BẦNG
}

\author{
Lâm Hoàng Chương* \\ Khoa Khoa học Tư nhiên, Trường Đại họ Cần Tho \\ *Ngưòi chịu trách nhiệm về bài viết: Lâm Hoàng Chuoong (email: lhchuong@ctu.edu.vn)
}

\section{Thông tin chung:}

Ngày nhận bài: 09/09/2020

Ngày nhận bài sưa: $27 / 10 / 2020$

Ngày duyệt đăng: 28/04/2021

Title:

Law of large number in the unfair game model

\section{Tù khóa:}

Bước đi ngẫu nhiên, luật số lớn, mô hình trò choi không công bằng, phưong pháp moment, toán tủ Markov

\section{Keywords:}

Law of large number, Markov operator, method of moment, unfair game model, random walk

\section{GIỚI THIẸU}

Trong lý thuyết xác suất, luật số số lớn chỉ ra rằng: khi ta chọn ngẫu nhiên một mẫu trong quần thể mà kích thước mẫu càng lớn thì các đặc trưng của mẫu càng "gần" với các đặc trưng của tổng thể. Trong thực tế, ta thấy rằng nhận định này cũng đúng trong rất nhiều lĩnh vực từ sinh học, nông nghiệp, kinh tế xã hội cho đến khoa học công nghệ. Đặc biệt, lý thuyết trò chơi với nhiều may rủi cũng cho ta nhiều kết quả liên quan đến luật số lớn.

Giả sử một trò chơi có hai người chơi là $\mathrm{A}$ và $\mathrm{B}$ thi đấu với nhau với 3 khả năng thắng, hòa và thua. Nếu xác suất thắng bằng xác suất thua thì ta gọi là trò chơi công bằng, còn ngược lại xác suất thắng và thua khác nhau thì gọi là trò chơi không công bằng. Chẳng hạn xét trò chơi tung 1 đồng $\mathrm{xu}$ : ông $\mathrm{A}$ thắng 1 đồng nếu đồng xu ra mặt sấp và thua 1 đồng nếu đồng xu ra mặt ngửa. Đây là mô hình trò chơi công bằng và ta biết rằng trung bình mỗi lần chơi thì người chơi hòa vốn. Kết quả này đã được chứng minh trong bài báo (Lâm Hoàng Chương và Dương Thị Bé Ba, 2017). Do đó, trong phạm vi bài báo này chúng ta tập trung nghiên cứu mô hình của một trò chơi của hai đối thủ. Ta xét trò chơi tung 1 con xúc xắc rất đơn giản: ông $\mathrm{A}$ thắng 1 đồng nếu xúc xắc ra mặt 2 chấm hoặc 4 chấm, hòa nếu xúc xắc ra mặt lẻ và thua 1 đồng nếu xúc xắc ra mặt 6 chấm. Đây còn được là mô hình trò chơi không công bằng được mô tả về mặt toán học như sau: ông $\mathrm{A}$ có số vốn ban đầu là $x$ đồng và sau mỗi ván chơi thì

- thắng 1 đồng với xác suất $1 / 3$,

- hòa vốn với xác suất $1 / 2$,

- thua 1 đồng với xác suất $1 / 6$.

Gọi $X_{n}$ (đồng) là số tiền của ông $\mathrm{A}$ sau ván thứ $n(n \geq 0)$ và không mất tính tổng quát ta giả sử 
$X_{0}=0$ là số vốn ban đầu của người chơi. Khi đó tập giá trị của $X_{n}$ là tập số nguyên $\mathbb{Z}$. Đây là mô hình bước đi ngẫu nhiên không cân bằng trên không gian trạng thái một chiều có cường độ dịch chuyển sang phải hoặc sang trái 1 đơn vị hoặc không dịch chuyển là khác nhau. Trường hợp xác suất thắng và thua như nhau thì đó là mô hình của bước đi ngẫu nhiên cân bằng. Các xác suất chuyển của bước đi ngẫu nhiên tại vị trí bất kỳ $k \in \mathbb{Z}$ ở thời điểm $n \geq 0$ được cho bởi các biểu thức sau:

$$
\begin{aligned}
& \mathbb{P}\left\{X_{n+1}=k+1 \mid X_{n}=k\right\}=1 / 3, \\
& \mathbb{P}\left\{X_{n+1}=k \mid X_{n}=k\right\}=1 / 2, \\
& \mathbb{P}\left\{X_{n+1}=k-1 \mid X_{n}=k\right\}=1 / 6 .
\end{aligned}
$$

Toán tử Markov $P$ tương ứng với bước đi ngẫu nhiên trên là $f \mapsto P f$ được xác định bởi

$$
P f\left(X_{n}\right)=\mathbb{E}\left[f\left(X_{n+1}\right) \mid X_{n}\right], \forall n \geq 0
$$

trong đó, $f$ là hàm đo được, bị chặn trên không gian trạng thái của bước đi ngẫu nhiên là tập số nguyên $\mathbb{Z}$. . Bằng các tính toán liên quan kỳ vọng có điều kiện, với mô hình của bước đi đang xét, ta luôn có

$$
P f(k)=\frac{1}{6}[f(k-1)+3 f(k)+2 f(k+1)] .
$$

Các tài liệu (Norris, 1998) và (Ross, 2010) trình bày khá chi tiết các tính chất của bước đi ngẫu nhiên trong không gian một chiều.

Thời gian gần đây, Lâm Hoàng Chương và ctv. (2017) đã đưa ra mô hình bước đi ngẫu nhiên chỉ có dịch chuyển sang phải (thắng) 1 đơn vị với xác suất $\alpha /(\alpha+\beta)$ và sang trái (thua) 1 đơn vị với xác suất với xác suất $\beta /(\alpha+\beta)$ là khác nhau. Khi đó một dạng của luật số lớn được chỉ ra đó là

$$
\frac{X_{n}}{n} \stackrel{P}{\longrightarrow} G=\frac{\alpha-\beta}{\alpha+\beta},
$$

khi $n \rightarrow+\infty$. Trong biểu thức trên, $\stackrel{P}{\longrightarrow}$ ký hiệu cho hội tụ theo xác suất của các biến ngẫu nhiên. Ở đó các tác giả đã xấp xỉ các moment bậc $k=1,2$ của $x_{n}$ để có giới hạn hội tụ đến các hằng số $G, G^{2}$.

Như vậy mô hình trong bài báo chúng ta đang xét tổng quát hơn trong Lâm Hoàng Chương và ctv. (2017) và ngoài ra các moment bậc $k=1,2$ của $X_{n}$ sẽ được tính ra kết quả cụ thể mà không cần thông qua việc dùng phương pháp xấp xỉ. Ta cũng có một dạng của luật số lớn trong mô hình trò chơi không công bằng (thắng - hòa - thua) như sau:

Định lý 1.1 Cho $\left(X_{n}\right)_{n \geq 0}$ là bước đi ngẫu nhiên không cân bằng như trên. Khi đó ta có

$$
\frac{X_{n}}{n} \stackrel{P}{\longrightarrow} \frac{1}{6} \text {, khi } n \rightarrow+\infty \text {. }
$$

Cấu trúc của bài báo được sắp xếp như sau. Mục 2 trình bày phương pháp chứng minh được sử dụng trong bài báo. Chứng minh chi tiết Định lý 1.1 được đưa ra ở Mục 3. Cuối cùng là phần kết luận vấn đề ở Mục 4.

\section{PHƯƠNG PHÁP NGHIÊN CÚU}

Một trong những phương pháp dùng để nghiên cứu luật số lớn được sử dụng rộng rãi thời gian gần đây là phương pháp moment. Theo đó, để chứng minh sự hội tụ của các biến ngẫu nhiên theo xác suất đến một hằng số, ta chứng minh sự hội tụ của dãy các moment của biến ngẫu nhiên đó. Pafnuty Chebyshev là người đầu tiên đã đề xuất phương pháp này trong nghiên cứu của ông để chứng minh định lý giới hạn trung tâm (Billingsley, 1995). Phương pháp này tiếp tục được phát triển trong nghiên cứu sự hội tụ của bước đi ngẫu nhiên trong môi trường ngẫu nhiên ở các công trình của (Depauw \& Derrien 2009) và (Lam, 2014). Phương pháp moment áp dụng cho luật số lớn trong bài báo này được phát biểu dưới dạng hệ quả như sau

Định lý 2.1 (Billingsley, 1995) Cho $\left(Z_{n}\right)_{n \geq 1}$ là các biến ngẫu nhiên cùng xác định trên một không gian xác suất và $a$ là một hằng số. Nếu $\lim _{n \rightarrow \infty} \mathbb{E}\left(Z_{n}\right)=a$ và $\lim _{n \rightarrow \infty} \mathbb{E}\left(Z_{n}{ }^{2}\right)=a^{2}$ thì $Z_{n}$ hội tụ theo xác suất đến $a$ khi $n \rightarrow \infty$.

Trong phần tiếp theo, ta sẽ vận dụng định lý 2.1 cho $Z_{n}=X_{n} / n$ và hằng số $a=1 / 6$. Khi đó ta cần chỉ ra rằng

$$
\lim _{n \rightarrow \infty} E\left(\frac{x_{n}}{n}\right)=\frac{1}{6} \text {, và } \lim _{n \rightarrow \infty} E\left[\left(\frac{x_{n}}{n}\right)^{2}\right]=\left(\frac{1}{6}\right)^{2} \text {. }
$$

\section{KẾT QUẢ THỰC HIÊN}

Ta chia bài toán làm 2 trường hợp cho moment bậc 1 và moment bậc 2 của $X_{n} / n$. Theo Lâm 
Hoàng Chương và ctv. (2017), các moment này chỉ tính xấp xỉ và lấy giới hạn khi $n \rightarrow \infty$. Ở đây chúng ta sẽ đưa ra biểu thức tính cụ thể cho các giá trị này, từ đó dễ dàng lấy giới hạn khi $n \rightarrow \infty$. Khi đó định lý 1.1 hoàn toàn được chứng minh nhờ áp dụng phương pháp moment như đã giới thiệu trong Định lý 2.1 .

Đầu tiên, ta xét trường hợp moment bậc 1 của $X_{n} / n$. Ta giải phương trình Poisson tương ứng với toán tử Markov $P$ của quá trình đã cho. Đây là một dạng phương trình vi phân tuyến tính của $f_{1}(m)$ xác định trên tập số nguyên $\mathbb{Z}$ nên nó có thể được giải bằng cách đệ quy theo biến $m \in \mathbb{Z}$. Sau đó, dựa vào tính chất nghiệm tìm được và kết hợp kỳ vọng có điều kiện để có kết quả mong muốn.

Mệnh đề 3.1 Moment bậc 1 của bước đi ngẫu nhiên đã cho là

$$
E\left(X_{n}\right)=\frac{1}{6} n \text {. Từ đó, ta có } \lim _{n \rightarrow \infty} E\left(\frac{X_{n}}{n}\right)=\frac{1}{6} \text {. }
$$

Chứng minh. Phương trình Poisson với $I$ là toán tử đồng nhất

$$
\left\{\begin{array}{c}
(P-I) f_{1}(m)=1, \forall m \in \mathbb{Z} \\
f_{1}(0)=0
\end{array}\right.
$$

luôn có nghiệm. Thật vậy, từ phương trình

$$
(P-I) f_{1}(m)=1
$$

dẫn đến

$$
2\left[f_{1}(m+1)-f_{1}(m)\right]-\left[f_{1}(m)-f_{1}(m-1)\right]=6 .
$$

Chia hai vế cho 2 ta được biểu thức tương đương

$$
\left[f_{1}(m+1)-f_{1}(m)\right]-\frac{1}{2}\left[f_{1}(m)-f_{1}(m-1)\right]=3 .
$$

Đệ quy theo $m, m-1, \ldots,-\infty$ ta được

$$
f_{1}(m)-f_{1}(m-1)=3 .\left[1+\frac{1}{2}+\frac{1}{2^{2}}+\ldots\right]=6 .
$$

Nếu $m \geq 1$ thì tiếp tục đệ quy theo $m-1, m-2, \ldots, 1$ và kết hợp giả thiết $f_{1}(0)=0$ ta được

$$
f_{1}(m)=6 m .
$$

Nếu $m \leq-1$ thì đệ quy theo $m, m+1, \ldots,-1$ ta cũng được kết quả tương tự. Như vậy

$$
f_{1}(m)=6 m, m \in \mathbb{Z}
$$

là một nghiệm của phương trình (3.1). Mặt khác, thế $m$ bởi $X_{n}$ trong biểu thức (3.1) rồi lấy kỳ vọng ta được

$$
E\left[f_{1}\left(X_{n+1}\right)\right]-E\left[f_{1}\left(X_{n}\right)\right]=1, \forall n \geq 0 .
$$

Trong biểu thức trên ta có sử dụng kết quả $E\left[E\left(f_{1}\left(X_{n+1}\right) \mid X_{n}\right)\right]=E\left[f_{1}\left(X_{n+1}\right)\right]$ được áp dụng từ tính chất kỳ vọng có điều kiện $E[E(X \mid Y)]=E[X]$ với mọi biến ngẫu nhiên $X$ và $Y$. Tiếp theo đệ quy theo $n, n-1, \ldots, 0$ ta được

$$
E\left[f_{1}\left(X_{n}\right)\right]=n, \forall n \geq 0 .
$$

Cuối cùng, kết hợp (3.2) và (3.3) ta được

$$
E\left[f_{1}\left(X_{n}\right)\right]=E\left[6 X_{n}\right]=n, \forall n \geq 0 .
$$

Từ đó ta có kết luận của Mệnh đề 3.1.

Đối với trường hợp moment bậc 2 thì ta cũng thực hiện các bước tương tự như moment bậc 1 . Tuy nhiên, trong phương trình Poisson ta sử dụng lại nghiệm $f_{1}(m)$ đã tìm được làm tham số. Khi đó nghiệm $f_{2}(m)$ được xây dựng dựa trên nghiệm $f_{1}(m)$ nên nó cũng có các tính chất của $f_{1}(m) C u ̣$ thể, ta có mệnh đề sau:

Mệnh đề 3.2 Moment bậc 2 của bước đi ngẫu nhiên đã cho là

$$
E\left(X_{n}^{2}\right)=\frac{1}{36} n^{2}-\frac{17}{36} n .
$$

Từ đó ta có

$$
\lim _{n \rightarrow \infty} E\left[\left(\frac{X_{n}}{n}\right)^{2}\right]=\frac{1}{36} .
$$

Chứng minh. Phương trình Poisson với $I$ là toán tử đồng nhất

$$
\left\{\begin{array}{c}
(P-I) f_{2}(m)=f_{1}(m), \forall m \in \mathbb{Z} \\
f_{2}(0)=0
\end{array}\right.
$$

luôn có nghiệm. Thật vậy, từ phương trình

$$
(P-I) f_{2}(m)=f_{1}(m)=6 m
$$

dẫn đến 
$2\left[f_{2}(m+1)-f_{2}(m)\right]-\left[f_{2}(m)-f_{2}(m-1)\right]=36 m$.

Chia hai vế cho 2 ta được biểu thức tương đương $\left[f_{2}(m+1)-f_{2}(m)\right]-\frac{1}{2}\left[f_{2}(m)-f_{2}(m-1)\right]=18 m$.

Đệ quy theo $m, m-1, \ldots,-\infty$ ta được

$$
\begin{aligned}
& f_{1}(m)-f_{1}(m-1) \\
& =18 \cdot\left[1(m-1)+\frac{1}{2}(m-2)+\frac{1}{4}(m-3)+\ldots\right] \\
& =18\left[\sum_{i=0}^{+\infty} \frac{1}{2^{i}}(m-(i+1))\right] .
\end{aligned}
$$

Ta phân tích biểu thức trong dấu ngoặc cuối cùng thành hai chuỗi số là chuỗi

$$
\sum_{i=0}^{+\infty} \frac{1}{2^{i}} m=2 m
$$

và chuỗi thứ hai

$$
\sum_{i=0}^{+\infty} \frac{1}{2^{i}}(i+1)=\frac{1}{(1-1 / 2)^{2}}=4 .
$$

Từ đó ta được

$$
f_{2}(m)-f_{2}(m-1)=36 m-72 .
$$

Nếu $m \geq 1$ thì tiếp tục đệ quy theo $m-1, m-2, \ldots, 1$ và kết hợp giả thiết $f_{2}(0)=0$ ta được

$$
f_{2}(m)=18 m(m+1)-72 m=18 m^{2}-54 m .
$$

Nếu $m \leq-1$ thì đệ quy theo $m, m+1, \ldots,-1$ ta cũng được kết quả tương tự. Như vậy

$$
f_{2}(m)=18 m^{2}-54 m, m \in \mathbb{Z}
$$

là một nghiệm của phương trình (3.4). Mặt khác, thế $m$ bởi $X_{n}$ trong biểu thức (3.4) rồi lấy kỳ vọng ta được

$$
E\left[f_{2}\left(X_{n+1}\right)\right]-E\left[f_{2}\left(X_{n}\right)\right]=n, \forall n \geq 0 .
$$

Trong kết quả trên, ta có sử dụng kết quả $E\left[E\left(f_{2}\left(X_{n+1}\right) \mid X_{n}\right)\right]=E\left[f_{2}\left(X_{n+1}\right)\right] \quad$ được áp dụng từ tính chất kỳ vọng có điều kiện $E[E(X \mid Y)]=E[X]$ với mọi biến ngẫu nhiên $X$ và $Y$. Tiếp theo đệ quy theo $n, n-1, \ldots, 0$ ta được

$$
E\left[f_{2}\left(X_{n}\right)\right]=\frac{(n-1) n}{2}, \forall n \geq 1 .
$$

Cuối cùng, kết hợp (3.5) và (3.6) ta được

$$
E\left[f_{2}\left(X_{n}\right)\right]=E\left[18 X_{n}^{2}-54 X_{n}\right]=\frac{(n-1) n}{2}, \forall n \geq 0 \text {. }
$$

Sử dụng kết quả ở Mệnh đề 3.1 ta có $E\left[X_{n}^{2}\right]=\frac{1}{18}\left[9 n+\frac{(n-1) n}{2}\right]=\frac{1}{36} n^{2}-\frac{17}{36} n, \forall n \geq 0$.

Kết quả được viết lại như sau

$$
E\left[\left(\frac{X_{n}}{n}\right)^{2}\right]=\frac{1}{36}-\frac{17}{36 n}, \forall n \geq 1 .
$$

Từ đó ta có kết luận của Mệnh đề 3.2. $\square$

Chú ý 3.3 Nếu ta đặt

$$
d(n)=\left|E\left(\frac{X_{n}}{n}\right)-\frac{1}{6}\right|+\left|E\left[\left(\frac{X_{n}}{n}\right)^{2}\right]-\frac{1}{36}\right|
$$

thì ta có tốc độ hội tụ trong Định lý 1.1 là

$$
d(n)=O\left(\frac{1}{n}\right)
$$

\section{KẾT LUẦN}

Bài báo đã chứng minh sự tồn tại một dạng của luật số lớn cho mô hình trò chơi không công bằng thông qua việc sử dụng phương pháp moment. Ngoài ra, điểm mấu chốt trong bài toán này ở chỗ ta có thể giải được phương trình Poisson tương ứng với toán tử Markov $P$. Từ đó có thể tính được moment của biến ngẫu nhiên một cách chi tiết và ngắn gọn hơn rất nhiều so với việc dùng phương pháp xấp xỉ. Ngoài ra, chúng ta có thể tính được các moment có bậc cao hơn. Chúng tôi kỳ vọng phương pháp này có thể được áp dụng cho các bài toán khác có liên quan.

\section{TÀI LIỆU THAM KHẢO}

Billingsley, P. (1995). Probability and measure. Wiley. New York.

Depauw, J., \& Derrien, J. M. (2009). Variance limite d'une marche aléatoire réversible en milieu aléatoire sur Z. Comptes Rendus Mathematique, 347(7-8): 401-406.

Lam, H. C. (2014). A quenched central limit theorem for reversible random walk in random 
environment on Z. Journal of Applied Probability, 51(4): 1051-1064.

Lâm Hoàng Chương, Lê Nguyễn Thúy Vân \& Dương Thị Tuyền. (2017). Luật số lớn cho bước đi ngẫu nhiên trong trường hợp một chiều. Tạp chi Khoa hoc Truòng Đại hoc Cần Tho, 52a: 17-21.

Lâm Hoàng Chương \& Dương Thị Bé Ba. (2017).

Tốc độ hội tụ trong định lý giới hạn trung tâm cho bước đi ngẫu nhiên trong một chiều. Tạp chi Khoa học Truờng Đại học Cần Tho, 49a: 73-78.

Norris J.R. (1998). Markov chains. Cambridge University Press.

Ross S. M. (2010). Introduction to Probability Models. Elsevier Inc. 